\title{
Acceso vascular en apoyo renal agudo
}

\author{
Armando Vázquez-Rangel \\ Departamento de Nefrología, Instituto Nacional de Cardiología Ignacio Chávez, Ciudad de México, México
}

\section{Resumen}

El acceso vascular es el primer eslabón para la terapia de apoyo extracorpórea renal. A diferencia de la terapia de reemplazo renal para pacientes con enfermedad renal crónica donde la fístula arteriovenosa es la primera opción, seguida del catéter tunelizado, en pacientes con lesión renal aguda el acceso vascular de elección es el catéter temporal. El presente estudio constituye una revisión narrativa de resumen de los estudios que analizan la elección, colocación y cuidados del acceso vascular temporal para apoyo renal agudo. En pacientes que cuentan con fístula arteriovenosa preexistente, su punción en pacientes con inestabilidad hemodinámica conlleva riesgos y no puede ser recomendada. El uso del catéter tunelizado de primera intención implica una destreza, tiempo y costos que limitan igualmente su recomendación. El cambio de catéteres por guía metálica ha mostrado ser un método seguro en ausencia de infección del catéter a retirar. El acceso femoral ha mostrado no ser inferior al acceso yugular derecho, mientras el acceso yugular izquierdo reduce la vida del circuito y debe ser considerado como cuarta opción después del yugular derecho, el femoral derecho y el femoral izquierdo. La longitud del acceso debe evaluarse de acuerdo con el sitio de inserción, mientras que los catéteres de $20 \mathrm{~cm}$ suelen ser adecuados para punción de la yugular derecha, la yugular izquierda suele requerir $25 \mathrm{~cm}$ para su llegada a la aurícula derecha. Por su parte, el acceso femoral incrementa su desempeño con longitudes de 25 a $35 \mathrm{~cm}$. La punción yugular posterior baja derecha es considerada una técnica de abordaje sencilla, segura y que permite una aproximación de hasta $5 \mathrm{~cm}$ más caudal hacia la aurícula derecha. La interacción entre catéteres centrales debe ser anticipada o evaluada ante el riesgo de aspiración de infusiones por el circuito extracorpóreo. En el cuidado del catéter, la aplicación de heparina 1,000 U/ml reduce el riesgo de sangrado al sellar los puertos comparada con heparina 5,000 U/ml. Por último, se presenta información sobre la elección de dispositivos de cobertura, el método de fijación, los materiales de recubrimiento de catéteres y el empleo de antibióticos, incluyendo las posturas de las guías del Kidney Dialysis Improving Global Outcomes (KDIGO) y la European Renal Best Practice (ERBP), además de la información disponible en el consenso del Acute Dialysis Quality Initiative, revisiones sistemáticas, metaanálisis y ensayos clínicos controlados.

PALABRAS CLAVE: Catéteres venosos centrales. Lesión renal aguda. Terapia de reemplazo renal.

\begin{abstract}
Vascular access is the first link for renal extracorporeal support therapy. Unlike renal replacement therapy for patients with end-stage renal disease where the arteriovenous fistula is the first option, followed by the tunneled catheter, in patients with acute kidney injury a temporary catheter is the first choice. The present article constitutes a narrative review summarizing the studies that analyze the choice, placement and care of temporary vascular access for acute renal support. In patients who have a pre-existing arteriovenous fistula, their puncture in patients with hemodynamic instability carries risks and cannot be recommended. The use of tunneled catheter as first option implies a complex, time-consuming procedure with higher cost that also limits its recommendation. The change of catheters by metal guide has shown to be a safe method in the absence of infection. Femoral access has been shown not to be inferior to right jugular access, while left jugular access reduces the life of the circuit and should be considered as a fourth option after right jugular, right femoral and left femoral sites. The length of
\end{abstract}

Correspondencia:

Armando Vázquez-Rangel

E-mail: drarmandovazquez@ hotmail.com
Fecha de recepción: 30-11-2017

Fecha de aceptación: 15-03-2017

DOI: 10.24875/GMM.M18000064
Gac Med Mex. 2018;Supp 1:22-30

Disponible en PubMed

www.gacetamedicademexico.com 
the access should be evaluated according to the insertion site, while $20 \mathrm{~cm}$ catheters are usually suitable for right jugular puncture, the left jugular usually requires $25 \mathrm{~cm}$ for achieving the position inside the right atrium. On the other hand, the femoral access increases its performance with lengths of 25-35 cm. The low posterior jugular approach is considered a simple, safe technique that allows up to $5 \mathrm{~cm}$ further towards the right atrium. The interaction between central catheters must be anticipated or evaluated because of the risk of aspiration of infusions by the extracorporeal circuit. The use of heparin 1000 $\mathrm{U} / \mathrm{ml}$ for sealing the ports reduces the risk of bleeding compared to heparin $5000 \mathrm{U} / \mathrm{ml}$. The reasoning on the evolution of catheters in structural coating materials, the decision on using a suture, the differences among dressings or securement devices, and the controversy over the use of antibiotics for sealing ports and for dressings are also discussed. The review includes the positions of the guidelines by the Kidney Dialysis Improving Global Outcomes and the European Renal Best Practice, in addition to the information available mainly on the consensus of the acute dialysis quality initiative, systematic reviews, meta-analysis and controlled clinical trials.

KEY WORDS: Central venous catheters. Acute kidney injury. Renal replacement therapy.

\section{Introducción}

El acceso vascular corresponde al primer paso dentro de la prescripción de la terapia de apoyo renal extracorpórea aguda. Es por sí mismo un procedimiento que confiere riesgos, y constituye además una de las principales causas de falla de la terapia de apoyo renal aguda, por lo que determina en gran parte el éxito que el tratamiento pudiese obtener en términos de metas alcanzadas y medidas de calidad durante el seguimiento de la terapia de apoyo renal aguda, incluyendo dosis alcanzada y vida del circuito extracorpóreo. En el presente artículo se ofrece una revisión narrativa de puntos específicos en la toma de decisiones sobre elección, colocación, monitoreo y cuidados del acceso vascular en apoyo renal agudo, dando prioridad a la literatura más reciente proveniente de guías de práctica clínica, consensos, revisiones sistemáticas, metaanálisis y ensayos clínicos controlados y aleatorizados (ECCA).

\section{Tipo de acceso vascular}

A diferencia de lo recomendado para pacientes en terapia de reemplazo renal crónica, donde las guías apoyan la construcción de fístulas arteriovenosas (FAV) como primer opción a fin de reducir complicaciones infecciosas e inflamación, y aumentar la eficiencia dialítica mediante flujos más altos, en el caso de apoyo renal agudo no se recomienda su uso por diversos motivos: 1) el requerimiento de apoyo inmediato una vez que se identifica el caso no permite un tiempo de espera para creación, maduración y uso de una FAV, inclusive si ello considerara la implantación de un injerto vascular; 2) los flujos sanguíneos requeridos en soporte renal agudo son inferiores al paciente crónico, ya sea por evitar desequilibrio electrolítico u osmótico, o porque los sistemas de apoyo prolongado o continuo no dependen del flujo, sino del tiempo para su eficiencia; 3) en pacientes crónicos portadores de fístula arteriovenosa, la instalación de agujas en caso de inestabilidad hemodinámica puede conllevar riesgo de trauma y trombosis, con la subsecuente pérdida de este valioso acceso; y 4) para terapias prolongadas o continuas, la dedicación requerida al cuidado del sitio de inserción de agujas es mucho mayor, ante el riesgo de fijación insuficiente y salida inadvertida del vaso con el subsecuente sangrado del sitio.

En el caso de catéteres tunelizados, si bien han mostrado una reducción significativa en la tasa de infecciones en pacientes en terapia de reemplazo renal crónica, su instalación implica una labor de mayor demanda técnica y consumo de tiempo, lo que en pacientes críticamente enfermos con fallo multiorgánico y posiblemente mayor riesgo de sangrado es difícil de justificar, además del costo mayor del catéter tunelizado. Las Guías en Lesión Renal Aguda de la $\mathrm{KDIGO}^{1}$ y la postura del grupo $\mathrm{ERBP}^{2}$ recomiendan iniciar terapia de apoyo renal en pacientes con lesión renal aguda con catéteres no tunelizados, con un nivel de recomendación 2D. En este sentido, y ante la meta de lograr recuperación de la función renal en los pacientes con lesión renal aguda, el catéter tunelizado sería reservado para aquellos pacientes agudos quienes después de un seguimiento intrahospitalario no lograran recuperación renal, planteando el cambio del catéter temporal por el tunelizado como un procedimiento electivo y no de urgencia.

El enfoque de cuándo migrar hacia un catéter tunelizado podría ser tomado a partir de la temporalidad de las bacteriemias asociadas a catéteres temporales. 
Oliver, et al. reportaron un seguimiento prospectivo en 218 pacientes con colocación de catéteres temporales, aunque incluyendo tanto pacientes crónicos como agudos, y encontrando una incidencia de bacteriemia en catéteres femorales del $3.1 \%$ en la primera semana y que ascendió al $10.7 \%$ a la segunda semana; en cambio, en catéteres yugulares la incidencia hasta la tercera semana fue del $5.4 \%$, ascendiendo en la cuarta semana al $10.3 \%^{3}$. Con ello, el retiro o cambio de un catéter temporal podría considerarse antes de la cuarta semana para catéteres yugulares y antes de la segunda semana para catéteres femorales, no obstante, en los protocolos internos de control y prevención de infecciones dentro de las Unidades de Cuidados Intensivos deberán seguirse haciendo cambio de catéteres; en nuestra Institución, por ejemplo, en tiempos no mayores a dos semanas.

\section{Procedimiento de colocación del acceso vascular}

El procedimiento de cambio de catéter temporal por tunelizado fue analizado por Casey, et al., quienes en 329 pacientes con 408 catéteres encontraron una tasa de bacteriemias de 3.0 por 1,000 catéteres-día en caso de colocación por nueva punción vs. 2.8 por 1,000 catéteres-día en caso de cambio de catéteres por guía metálica en el mismo sitio, concluyendo que este procedimiento es seguro y con menores complicaciones potenciales asociadas dada su naturaleza ${ }^{4}$. De igual forma, la colocación de un nuevo catéter temporal podría llevarse a cabo a través de una guía metálica a partir de un acceso venoso central previo, evitando así la técnica de punción. Coupez, et al. tomaron 178 pacientes procedentes de una muestra de pacientes incluidos en un ECCA previo, encontrando un porcentaje de colonización igual para ambas técnicas (5.6\%), aunque el riesgo de disfunción del acceso fue mayor en el caso de colocación por guía metálica vs. por punción (razón de tasas de riesgo 0 hazard ratio [HR]: 3.67 [2.07 $\div 6.49])^{5}$. De esta forma, en pacientes de alto riesgo de complicaciones por punción, el cambio del acceso por guía metálica es considerada una técnica segura.

En caso de requerirse colocación por punción, el empleo de ultrasonido en tiempo real para dirigir el procedimiento ha mostrado una reducción en la incidencia de complicaciones. En el metaanálisis de Rabindranath, et al. que incluyó 7 ECCA para un total de 830 catéteres, el procedimiento dirigido por ultrasonido disminuyó el fallo en la colocación (riesgo relativo [RR]: $0.12[0.04 \div 0.37]$ ), el fallo del primer intento (RR: $0.4[0.29 \div 0.56])$, las punciones arteriales (RR: $0.22[0.06 \div 0.81]$ ) y la formación de hematoma (RR: 0.27 [0.08 $\div 0.88$ ); adicionalmente, disminuyó el tiempo requerido para canular la vena (diferencia media: -1.4 [-2.17 $\div-0.63]$ ) y el número de intentos para la inserción del catéter (diferencia media: $-0.35[-0.54 \div-0.16])^{6}$. Tanto las guías $\mathrm{KDIGO}^{1}$ como el grupo ERBP2 recomiendan el uso del ultrasonido durante la colocación del catéter con un nivel $2 \mathrm{~A}$, por supuesto entendiendo que su disponibilidad no es universal en todos los niveles de atención en salud, por lo que los centros académicos podrían aprovechar para que de forma supervisada se pueda fomentar el entrenamiento con y sin el uso de esta tecnología. En cuanto a la técnica ultrasonográfica, Tamman, et al. compararon la colocación por visualización en eje axial (fueran del plano) de la vena yugular interna contra visión longitudinal (en el plano), sin encontrar diferencia en tasa de éxito, tiempo de colocación del acceso, número de intentos, punción de carótida, hematoma, hemo o neumotórax, o infección ${ }^{7}$; por lo que la elección del eje de proyección es un tema personal ligado a la comodidad y experiencia del usuario.

\section{Sitio de colocación del acceso venoso}

La elección sobre el sitio de inserción del catéter para apoyo agudo ha cambiado a lo largo de los últimos años. Los programas de entrenamiento durante los estudios previos a la especialidad de Nefrología tradicionalmente enseñan la colocación de catéteres subclavios para acceso venoso central en general, no obstante, ya en 1991 Schillinger, et al. reportaron 100 pacientes sometidos a hemodiálisis en el periodo de 1986 a 1990, 50 de ellos mediante catéter subclavio y 50 mediante vena yugular interna, encontrando por estudio angiográfico un $42 \%$ de estenosis venosa en catéter subclavio vs. $10 \%$ en catéter yugular, con un tiempo promedio de seguimiento de $31 \pm 21.8$ y $31.7 \pm 16$ días respectivamente ${ }^{8}$. Dichos hallazgos han colocado al acceso subclavio como la última opción ante el riesgo de no recuperación de la función renal tras un evento agudo y por ende la necesidad de un acceso vascular en forma crónica, donde la posibilidad de instalación de fístula arteriovenosa se vería claramente reducida en caso de estenosis proximal de miembros torácicos.

En cuanto al comparativo entre acceso venoso yugular y femoral, Parienti, et al., en un ECCA (estudio 
Cathedia) de 750 pacientes en 12 centros hallaron un mayor porcentaje de hematomas en la colocación del acceso yugular ( 3.6 vs. $1.1 \%, p=0.03)^{9}$. El mismo grupo de autores reportaron en otra publicación resultados similares en cuanto a la tasa de reducción de urea en hemodiálisis intermitente $(50.8 \pm 16.1 \%$ en femoral vs. $52.8 \pm 15.8 \%$ en yugular, $p=0.30$ ), y en tiempo no efectivo de tratamiento durante terapia de apoyo continuo (1.17 [0.75 $\div 1.50] \mathrm{h}$ para ambos, $\mathrm{p}=0.98)^{10}$. Dugué, et al. en un subanálisis del estudio Cathedia en 134 pacientes, 57 asignados a inserción femoral y 77 a inserción yugular, encontraron que el tiempo requerido para la inserción femoral fue significativamente menor $(10[7 \div 15]$ vs. $12[8 \div 19] \mathrm{min}$, $p=0.01)$, al igual que menor porcentaje de falla en dicha colocación ( 2.3 vs. $12.8 \%, p=0.003$ ); las curvas de supervivencia libre de colonización bacteriana (HR: 0.99 [0.61 $\div 1.59], p=0.96$ ) y libre de disfunción de catéter (HR: 1.20 [0.74 $\div 1.95], p=0.46$ ) no mostraron diferencia entre los sitios de inserción ${ }^{11}$. Parienti, et al., en el estudio Cathedia ya citado, analizó los eventos de colonización de catéter durante su retirada al dividir a los pacientes por índice de masa corporal, encontrando que aquellos con un valor $>28.4 \mathrm{~kg} / \mathrm{m} 2$ mostraron una menor incidencia de colonización en el grupo asignado a catéter yugular vs. femoral (HR: 0.40 [0.23 $\div 0.69], p=0.001$ ); mientras que la probabilidad de colonización del catéter femoral incrementa en razón al índice de masa corporal, en el caso del acceso yugular no presenta relación entre ambas variables $^{9}$. Finalmente, Marik, et al. en una revisión sistemática de ECCA y estudios de cohorte con un total de 113,652 catéteres-día demostraron por meta regresión una interacción significativa entre el riesgo de infección y el año de publicación, y con ello una disminución progresiva en el riesgo de infección del acceso femoral en estudios incluidos desde 1998 al $2010^{12}$. Dado el menor número de complicaciones, la seguridad en el seguimiento reportada en estudios recientes, y la facilidad de la técnica femoral, el grupo internacional del Acute Dialysis Quality Initiave (ADQI) en la publicación de la conferencia 18 sugiere que, para la elección del sitio de acceso venoso para terapia de apoyo renal aguda, ambas yugular derecha o femoral derecha sean consideradas como primera opción en pacientes sin obesidad ${ }^{13}$.

Con respecto al acceso yugular, el lado y el abordaje anatómico ha mostrado diferencias importantes. Mathur, et al. en un ECCA asignaron a 104 pacientes a colocación de catéter yugular por abordaje posterior o abordaje central, presentando este último un mayor porcentaje de complicaciones relacionados al procedimiento incluyendo punción de carótida y neumotó$\operatorname{rax}(14.8$ vs. $6 \%, p=0.04$ ), no obstante, el abordaje posterior presentó mayor porcentaje de interrupciones en el tratamiento por obstrucción de flujo (46 vs. $9.25 \%, p<0.01)^{14}$. Si bien cada uno de los abordajes puede representar una ventaja dependiendo de la situación anatómica entre vena yugular interna y arteria carótida (Fig. 1, A y B). Una tercera opción que corresponde al abordaje posterior bajo (Fig. 1, C) podría reducir las complicaciones. En este tipo de acceso existe una distancia menor tanto en el trayecto interno en cuello como en longitud hacia la unión cavoatrial, con una diferencia entre $3-5 \mathrm{~cm}$ con respecto al abordaje posterior tradicional, y de esta manera se reducen los problemas de flujo, mejorando la comodidad en pacientes alertas, y facilitando los cuidados del catéter, lo que potencialmente reduce riesgo de colonización al tener una fijación cutánea baja. Con respecto al lado de colocación, Parienti, et al. encontraron que mientras la incidencia de disfunción del acceso no fue diferente entre femoral y yugular derecho ( 10.3 vs. $6.6 \%, p=0.09$ ), si lo fue significativamente entre femoral y yugular izquierdo (10.3 vs. $19.5 \%$ respectivamente, $p=0.02)^{10}$. Morgan, et al. también en un ECCA, evaluaron el desempeño de catéteres yugulares en apoyo extracorpóreo renal dependiendo la localización final de la punta, ya sea en la aurícula derecha o en la vena cava superior; para llevar a cabo lo anterior, asignaron a pacientes con acceso yugular derecho a recibir un catéter de $15 \mathrm{~cm}$ (corto) vs. $20 \mathrm{~cm}$ (largo), y a pacientes con acceso yugular izquierdo a catéter de $20 \mathrm{~cm}$ (corto) vs. $24 \mathrm{~cm}$ (largo). El grupo de catéteres largos se asoció a una mayor vida del circuito extracorpóreo $(24[11 \div 32]$ vs. $17.5[8 \div 23] h, p=0.001)$, mayor porcentaje de dosis entregada (91 [85 $\div 100]$ vs. $81[72 \div 87] \%, p=0.001$ ), menor número de filtros coagulados por paciente (2.3 vs. $3.6, p=0.04$ ) y menor número de filtros empleados en su estancia ( 3.5 vs. $5.0, p=0.03)^{15}$. Engstrom, et al., en un análisis retrospectivo de 532 catéteres yugulares, aunque tunelizados, observaron que aquellos con una posición final de la punta en atrio derecho no mostraban diferencia en la tasa de disfunción entre catéteres yugulares derechos vs. izquierdos, sin embargo, cuando la posición final de la punta era en vena cava superior o unión cavoatrial, los catéteres izquierdos tuvieron una tasa significativamente mayor de disfunción (0.25 vs. 0.11 eventos por 100 catéteres-días, $p=0.036)^{16}$. En conclusión, el acceso yugular izquierdo ofrece menor desempeño 

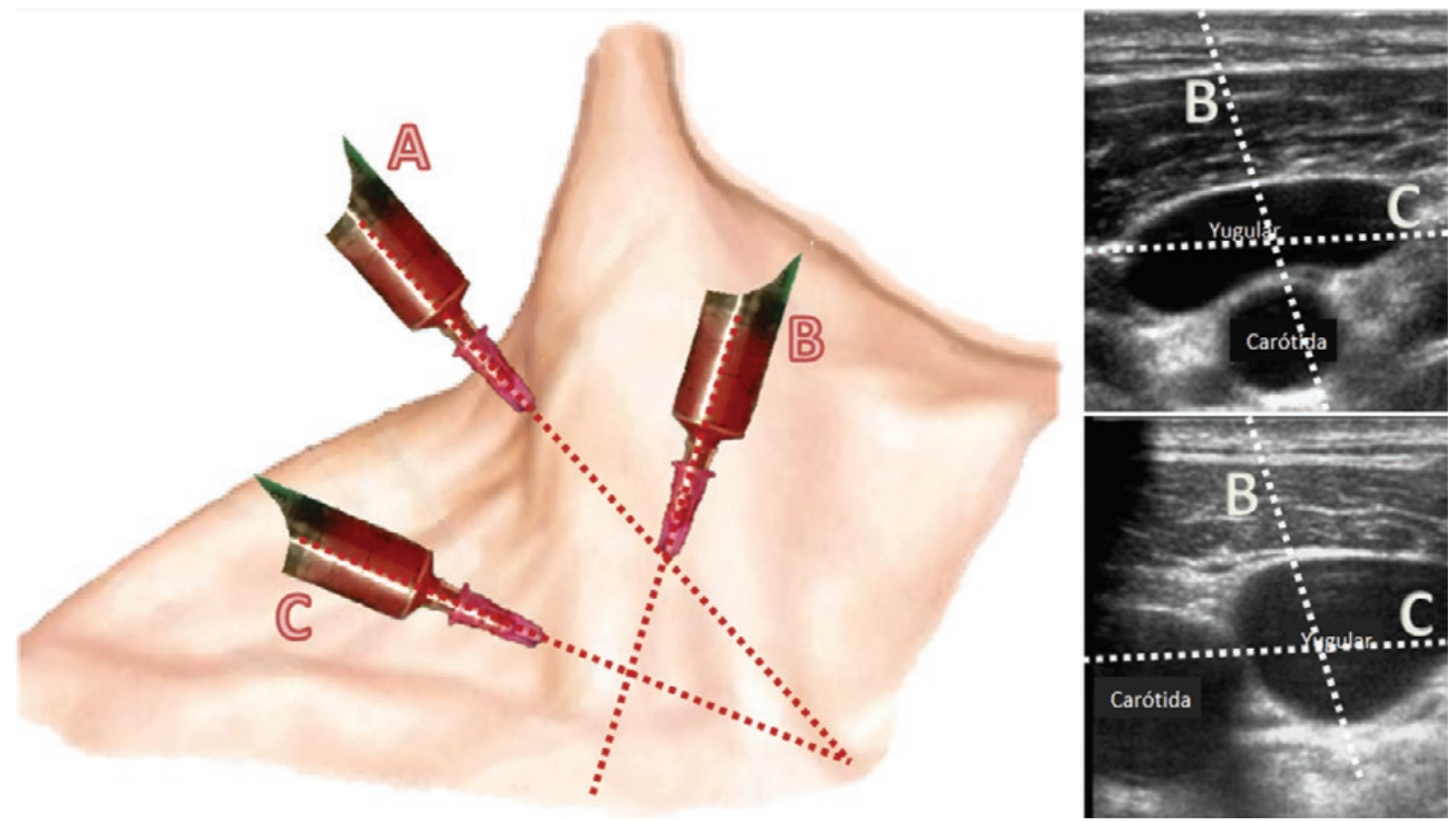

Figura 1. Abordajes para acceso yugular. A: La punción yugular posterior, por arriba del cruce de la vena yugular externa por el esternocleidomastoideo y dirigida al manguito del esternón, reduce complicaciones intratorácicas, pero tiene un trayecto cervical largo y una fijación cutánea alta. B: La punción yugular central o media, en el vértice de los fascículos anterior y posterior del esternocleidomastoideo y dirigida al pezón ipsilateral, aunque confiere menor comodidad, resulta sencilla y útil cuando la imagen ultrasonográfica ubica a la vena yugular lateral a la arteria carótida. C: La punción yugular posterior baja, 1-2 cm por arriba de la clavícula detrás del fascículo posterior del esternocleidomastoideo y dirigida al manguito del esternón, es sencilla, de bajas complicaciones, con fijación que deja libre el área cervical, y especialmente útil cuando la vena yugular por ultrasonido se ubica anterior a la arteria carótida.

comparado con el yugular derecho o el femoral, e inclusive con un potencial de estenosis en el segmento de vena innominada equivalente al acceso subclavio izquierdo. En caso de optar por colocación yugular izquierda, se deberá verificar la longitud adecuada del catéter para asegurar su llegada al atrio derecho, mientras que para el acceso yugular derecho, la posición superior a la unión cavoatrial no parece reducir su desempeño.

Un aspecto adicional sobre la posición de la punta del catéter de hemodiálisis es la potencial interacción con otros catéteres centrales empleados en la administración de soluciones, productos sanguíneos y fármacos, ya sea en infusión o en bolo. Kam, et al. publicaron un modelo simulando el vaso e inyectando tinta como marcador visual y creatinina como marcador cuantitativo; los marcadores fueron aspirados por el catéter de hemodiálisis a menos de que este se encontrara $2 \mathrm{~cm}$ por detrás del otro catéter central ${ }^{17}$. En la clínica, dicha aspiración puede tener traducción en escenarios de mayor inestabilidad hemodinámica durante la conexión 0 al subir flujo de bomba de sangre, en incrementos de presión del circuito asociado a transfusión de productos sanguíneos o en falla de metas de calcio ionizado durante anticoagulación con citrato por recirculación del calcio infundido, entre otros. Las opciones para contrarrestar dicho efecto de aspiración podrían incluir: 1) mantener la punta del acceso de hemodiálisis por detrás de la otra vía central, lo cual es solo posible en caso de acceso yugular derecho; 2) suponer que la aspiración de sangre mezclada en atrio derecho implicará una menor cantidad relativa de sustancias aspiradas, lo cual no ha sido evaluado; 3) modificar la posición del otro acceso central; 4) simplemente optar por acceso de hemodiálisis en posición femoral, o usar accesos de hemodiálisis trilumen, en los cuales la tercera vía es empleada para la administración específica de un fármaco, teniendo su salida por delante del orificio de aspiración de sangre (Fig. 2). La seguridad en el empleo de estos catéteres trilumen fue probada por Contreras, et al. en un ECCA multicéntrico en 485 pacientes, en el cual encontraron una tasa de incidencia de colonización de 12.4/1,000 catéteres-días 


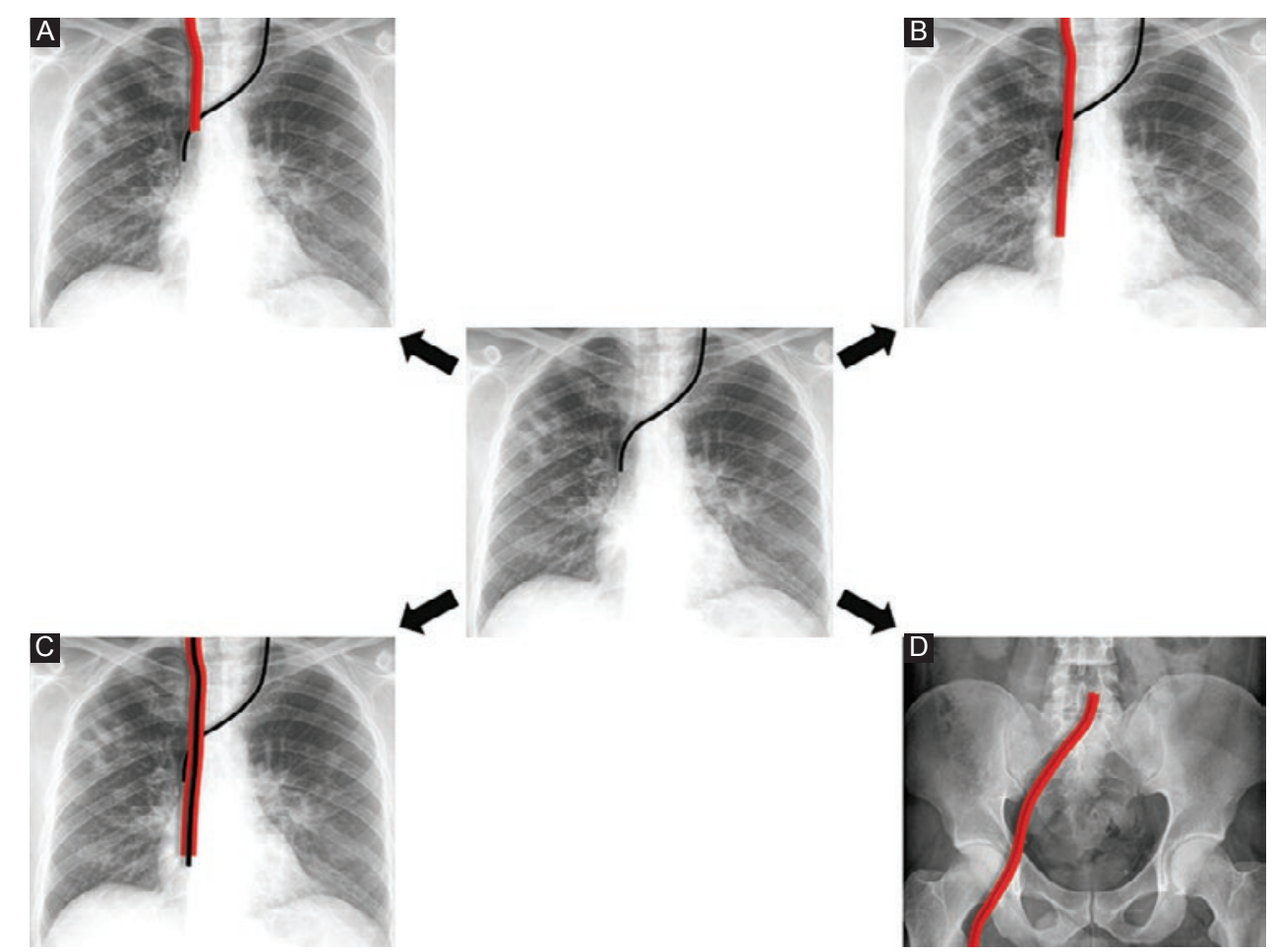

Figura 2. Interacción entre accesos venosos. En el caso de la preexistencia de un acceso yugular izquierdo central para administración de fármacos y soluciones, la colocación del catéter de hemodiálisis en yugular derecho permite en teoría dejar la punta apenas arriba del acceso previo para evitar aspiración de infusiones (A), o la introducción hasta atrio derecho bajo la teoría de menor aspiración relativa a la mezcla venosa (B), o la introducción de un catéter trilumen e infusión por tercer lumen de algún fármaco seleccionado (C), o finalmente optar por acceso femoral para evitar interacción (D).

con accesos bilumen vs. 10.2/1,000 catéteres-días con accesos trilumen $(p=0.59)$, y sin diferencia en los flujos alcanzados en sesiones de hemodiálisis 0 aféresis ${ }^{18}$.

En cuanto a accesos femorales, es reconocido el efecto de mayor recirculación de sangre y, por ende, reducción en la dosis entregada. LeBlanc, et al. en 1996 evaluaron pacientes que requerían apoyo renal agudo reportando recirculación de $16.1 \pm 9.1 \%$ en acceso femoral vs. $4.1 \pm 3.6 \%$ en acceso subclavio ( $p=0.001$ ); a destacar, al comparar la longitud del acceso femoral mostró recirculación de $22.8 \pm 9.1 \%$ con catéter de $13.5 \mathrm{~cm}$ vs. $12.6 \pm 6.9 \%$ en catéter de $19.5 \mathrm{~cm}(p=0.004)^{19}$. Un estudio similar por Little, et al. encontró recirculación en accesos temporales femorales de $13.1 \%$ vs. $0.4 \%$ en yugulares $(p=0.001)$; de igual forma, catéteres femorales de $<20 \mathrm{~cm}$ tuvieron recirculación del $26.3 \%$ comparada con un $8.3 \%$ en $>20 \mathrm{~cm}(p=0.007)^{20}$. En el estudio de Parienti, et al., aquellos pacientes con catéteres femorales de $20 \mathrm{~cm}$ mostraron una menor tasa de reducción de urea en comparación con acceso de $20 \mathrm{~cm}$, pero en posición yugular (-15.5 [-28.9 a -2.2], $\mathrm{p}=0.03)$; sin embargo, esta diferencia no se observó si el catéter femoral era de $25 \mathrm{~cm}^{10}$. Por su parte, Bellomo, et al., en un subanálisis del ECCA Randomized Evaluation of Normal vs. Augmented Level Renal Replacement Therapy (RENAL), analizó por regresión lineal múltiple la dosis entregada durante terapias de reemplazo renal continuas y encontró que el catéter femoral la reducía en $1.03 \%$, no obstante, un catéter de $13.5 \mathrm{Fr} o$ $24 \mathrm{~cm}$ aumentaba la dosis entregada en 4.2 y $1.88 \%$ respectivamente ${ }^{21}$. Finalmente, en el estudio de Kim, et al. en pacientes recibiendo terapia de apoyo renal continua con acceso femoral, la vida del circuito fue significativamente menor si el acceso era en el lado izquierdo vs. derecho (10.6 \pm 7.4 vs. $15.0 \pm 14.3$ horas respectivamente, $p=0.019)^{22}$. En conclusión, mientras que el acceso femoral derecho puede ser considerado como primera elección junto con yugular derecho, el femoral izquierdo quedaría como segunda prioridad; la recomendación en ambos lados sería una longitud de catéter idealmente de al menos $24 \mathrm{~cm}$.

\section{Características del acceso vascular}

Pocas características inherentes al catéter han mostrado una ventaja clínica dentro de la variedad 
existente. En cuanto a la conformación de la punta, las opciones disponibles incluyen como ejemplo puntas convencionales, puntas escalonadas, puntas separadas, etc., pudiendo tener además orificios únicos, múltiples o largos circunferenciales, entre otros, no obstante, existe poca evidencia sobre la ventaja de alguno de ellos sobre los demás. Con respecto al extremo para su conexión, las opciones pueden incluir extensiones rectas, extensiones curvas, o catéter precurvado. Weijmer, et al. evaluaron en forma prospectiva catéteres precurvados vs. extensiones rectas colocados por vía yugular, donde 15 vs. 53\% respectivamente debieron ser retirados por complicaciones (infección del sitio del inserción, bacteriemia asociada al catéter, problemas de flujo, retirada accidental o rotura del catéter) $(p<0.001)$, y donde los eventos de bacteriemia asociados a catéter fueron de 0 vs. $5.6 / 1,000$ catéteres-días $(p<0.01)^{23}$; la comodidad del sentido caudal de las extensiones y facilidad para los protocolos de cuidado son referidas por los autores como explicaciones para obtener mejores resultados, mientras que las extensiones rectas serían por mismas razones de elección para la posición femoral.

Por otro lado, algunas publicaciones sugieren que parte de las complicaciones asociadas a catéteres como trombosis o colonización son favorecidas por las irregularidades en la superficie de los materiales, abriendo la posibilidad a evaluar catéteres recubiertos. Verbeke, et al. analizaron por microscopia electrónica la integridad en la superficie de catéteres convencionales de hemodiálisis vs. catéteres recubiertos con un copolímero tras su uso en pacientes durante una semana; los autores reportaron $62.7 \pm 32.9 \times 10^{3}$ orificios $/ \mathrm{mm}^{2}$ demostrables en los primeros, interpretado como un desprendimiento del sulfato de bario contenido desde fabricación, mientras que los catéteres recubiertos no mostraron defectos cuantificables $^{24}$. Al incubar los catéteres in vitro con Staphylococcus epidermidis, los convencionales mostraron un rápido crecimiento bacteriano $(0.512 \% / 10 \mathrm{~min}) \mathrm{com}$ parado con los catéteres recubiertos $(-0.021 \% / 10 \mathrm{~min})$ $(p<0.001)^{24}$. Lia, et al., con la Colaboración Cochrane, publicó una revisión sistemática de ECCA sobre la recubierta o impregnación de catéteres, aunque no necesariamente en el contexto de hemodiálisis; se incluyeron 57 estudios con 16,784 catéteres y 11 tipos de impregnación ${ }^{25}$. En general, la impregnación redujo significativamente las bacteriemias relacionadas a catéter (número necesario para beneficio: 50 ), y colonización de catéter (número necesario para beneficio: 11), limitándose esta observación dentro de las
Unidades de Cuidados Intensivos; no se observó cambio en la incidencia de sepsis, mortalidad o infección del sitio de inserción ${ }^{25}$. Sin embargo, debido a la heterogeneidad de los estudios, su uso no puede ser recomendado en forma sistemática, sobre todo aqueIlos con antimicrobianos; y mientras otras variantes como las recubiertas con plata muestran resultados positivos, aún se requieren más estudios que confirmen su utilidad.

\section{Cuidados del acceso venoso}

Los cuidados internos en términos de patencia de los lúmenes y externos en términos de higiene son de gran importancia para mantener el acceso funcional y reducir las complicaciones derivadas de su uso. Con respecto a medidas de anticoagulación, Wang, et al. en un metaanálisis para Cochrane incluyeron 27 ECCA en 3,003 pacientes con enfermedad renal crónica recibiendo hemodiálisis; teniendo a heparina $5,000 \mathrm{UI} / \mathrm{ml}$ como solución para sellar el catéter como grupo control, solo el plasminógeno tisular recombinante (rt-PA) redujo en forma significativa la disfunción del acceso (RR: 0.58 [0.37 $\div 0.91]$ ), aunque derivado de un solo estudio ${ }^{26}$. A destacar, el uso de dosis baja de heparina no fue inferior a la dosis de $5,000 \mathrm{UI} / \mathrm{h}$ (RR: $0.90[0.10 \div 8.31])^{26}$. En este sentido, $\mathrm{Hu}$, et al. asignaron en un ECCA a 184 pacientes a sello de catéter con heparina 5,000 Ul/ml vs. 1,000 $\mathrm{UI} / \mathrm{ml}$, encontrando que tras $10 \mathrm{~min}$ del sello los valores de tiempo de tromboplastina activada en sangre periférica eran francamente más prolongados en la dosis mayor $(130.1 \pm 34.4$ vs. $32.2 \pm 5.0$ segundos, $p<0.05)$, además de mostrar en una segunda cohorte una mayor caída en los niveles de hematocrito (-2.30 \pm 1.73 vs. $-1.37 \pm 1.82 \%, p=0.04)$ y mayor incidencia de sangrado ( 9.5 vs. $1.1 \%, p=0.02$ ) en el grupo de $5,000 \mathrm{UI} / \mathrm{ml}^{27}$.

Con respecto al citrato concentrado para el sellar del acceso, en el metaanálisis de Wang, et al. no se evidenció una reducción en la disfunción del acceso con su uso, aunque si una reducción en la incidencia de bacteriemia asociada a catéter (RR: 0.49 [0.36 $\div$ $0.68])^{26}$; no obstante en contraste, en el metaanálisis de Zhao, et al. con 13 ECCA y 1,770 pacientes, el uso de citrato sin combinación con otro agente no mostró ventaja en la incidencia de bacteriemias vs. heparina (RR: 0.54 [0.22 $\div 1.3]$ ), e inclusive a menor concentración de citrato menor probabilidad de bacteriemia asociada a catéter (concentración de 30-46.7\%, RR: 0.5 [0.14 $\div 1.83], p=0.30$; concentración de 4.6-7\%, 
RR: 0.27 [0.12 $\div 0.64], p=0.003$; concentración de 1.04-4\%, RR: 0.38 [0.25 $\div 0.58], p<0.001)^{28}$. El riesgo de sangrado con citrato vs. heparina fue significativamente menor (RR: 0.48 [0.30 $\div 0.76], p=0.002)^{28}$.

En cuanto al empleo de soluciones con antibiótico para sellar el catéter, en efecto, el metaanálisis de Wang, et al. mostró una reducción en los eventos de bacteriemias (RR: $0.27[0.11 \div 0.70])^{26}$, aunque otros autores han expresado la preocupación de la exposición sistémica al antibiótico, ya sea por el potencial de inducir resistencia bacteriana o por sus efectos adversos. Dogra, et al., asignaron en un ECCA a pacientes con catéteres tunelizados a recibir sellado con citrato + gentamicina $(40 \mathrm{mg} / \mathrm{ml})$ vs. heparina, demostrando niveles de gentamicina previos a la hemodiálisis de $2.8 \mathrm{mg} / \mathrm{l}(0.6 \div 3.5)$ vs. $0.2 \mathrm{mg} / \mathrm{l}(0.2 \div 0.2)$ respectivamente $(p=0.008)$; a destacar, 4 de los 42 pacientes del grupo de gentamicina experimentaron mareo, inestabilidad, hipoacusia o ataxia ${ }^{29}$.

Como sugerencia, el uso de heparina a dosis baja $(1,000 \mathrm{UI} / \mathrm{ml})$ o citrato en concentración baja (1-7\%) deben ser consideradas como opciones que reducen el riesgo de sangrado manteniendo la funcionalidad del acceso. Finalmente, tanto las guías KDIGO ${ }^{1}$ como el grupo ERBP 2 plantean no usar sello con antibióticos en catéteres para apoyo agudo con un nivel de recomendación $2 \mathrm{C}$, dado el riesgo de exposición sistémica a dichos agentes en forma continua.

Por último, con respecto a los cuidados externos del acceso, Ullman, et al., en un metaanálisis tipo red para Cochrane, compararon los parches o dispositivos de cobertura de catéteres, incluyendo 22 ECCA con 7,436 pacientes. En sus resultados, las coberturas impregnadas con antibiótico redujeron significativamente la incidencia de bacteriemia asociada a catéter (RR: 0.6 [0.39 $\div$ 0.93]) en comparación con otras coberturas; en segundo lugar, las coberturas impregnadas con clorhexidina (comparadas con los parches de poliuretano convencionales) tuvieron menor número de bacteriemias por 1,000 pacientes-días (RR: 0.51 [0.33 $\div 0.78])$ y menor riesgo de colonización de la punta del catéter (RR: $0.58[0.47 \div 0.73])^{30}$. Los autores concluyen, además, que los dispositivos de apoyo sin sutura parecen ser la técnica más efectiva para reducir la incidencia de bacteriemia asociada a catéter $^{30}$. Mimoz, et al., en un ECCA, asignaron a 2,546 pacientes a recibir antisepsia con solución de alcohol-clorhexidina vs. alcohol-iodo-povidona, además de vs. sin fricción durante el procedimiento; en sus resultados, la solución con alcohol-clorhexidina se asoció con una menor incidencia de bacteriemia asociada a catéter (HR: 0.15 [0.05 $\div 0.41], p=0.002$ ) o colonización del catéter (HR: 0.18 [0.13 $\div 0.24]$, $p=0.001$ ), mientras que la fricción aplicada durante la antisepsia no mostró beneficio ${ }^{31}$. En conclusión, se sugiere la antisepsia con alcohol-clorhexidina y el uso de dispositivos de cobertura o fijación sin sutura e impregnados con clorhexidina en pacientes que así lo toleren, contemplando el riesgo de irritación cutánea. Nuevamente, tanto las guías KDIGO ${ }^{1}$ como el grupo ERBP $^{2}$ recomiendan no emplear dispositivos de cobertura impregnados con antibióticos, ante la necesidad de más estudios concernientes al riesgo de resistencia bacteriana.

\section{Conclusiones}

El acceso vascular constituye el punto inicial para la prescripción de la terapia de apoyo renal aguda, y por consiguiente de él depende en buena parte el éxito de dicho tratamiento. El catéter temporal es la forma recomendada de acceso venoso para pacientes en apoyo agudo y su colocación puede llevarse a cabo por cambio de un acceso previo por guía metálica o por punción idealmente guiada por ultrasonido. La planeación del acceso debe contemplar la posición de otros accesos venosos previos, a fin de reducir su interacción. Tanto el acceso yugular derecho como femoral derecho son la primera opción de sitio de inserción en pacientes no obesos, seguidos por el femoral izquierdo, el yugular izquierdo y, por último, la subclavia derecha e izquierda. La longitud del acceso deberá contemplar la llegada al atrio derecho para las inserciones yugulares, lo que implica generalmente $5 \mathrm{~cm}$ más en el lado izquierdo que en el derecho, mientras que la colocación yugular posterior baja para ambos lados puede mantener complicaciones reducidas y optimizar la posición de la punta y su funcionalidad por la longitud ahorrada. Para el acceso femoral, debe contemplarse un mínimo de $24 \mathrm{~cm}$. Tanto heparina a dosis baja $(1,000 \mathrm{Ul} / \mathrm{ml})$ como citrato a concentración baja (1-7\%) son opciones adecuadas para sellar los puertos del catéter. Como parte de los cuidados, los dispositivos de cobertura con clorhexidina y sin sutura parecen reducir la incidencia de infecciones. Son necesarios más estudios para establecer la seguridad del empleo de soluciones para sellar puertos o dispositivos de cobertura que contengan antibióticos y, en el futuro, el recubrimiento de catéteres podría mejorar su perfil funcional y de complicaciones. 


\section{Conflicto de intereses}

AVR ha participado como ponente para Baxter y Medtronic. La información escrita en el presente artículo representa su punto de vista personal y no proviene de otras instituciones, grupos o empresas.

\section{Financiación}

Armando Vázquez-Rangel ha sido apoyado por el fondo S0008-FONSEC SSA/IMSS/ISSSTE, Proyecto 234130 de CONACYT.

\section{Bibliografía}

1. KDIGO Clinical Practice Guideline for Acute Kidney Injury. Section 5: Dialysis Interventions for Treatment of AKI. Kidney Int Suppl. 2012;2(1):89-115.

2. Jörres A, John S, Lewington A, ter Wee PM, Vanholder R, Van Biesen W, et al. A European Renal Best Practice (ERBP) position statement on the Kidney Disease Improving Global Outcomes (KDIGO) Clinical Practice Guidelines on Acute Kidney Injury: part 2: renal replacement therapy. Nephrol Dial Transplant. 2013;28(12):2940-5

3. Oliver MJ, Callery SM, Thorpe KE, Schwab SJ, Churchill DN. Risk of bacteremia from temporary hemodialysis catheters by site of insertion and duration of use: a prospective study. Kidney Int. 2000;58(6):2543-5.

4. Casey J, Davies J, Balshaw-Greer A, Taylor N, Crowe AV, McClelland P. Inserting tunnelled hemodialysis catheters using elective guidewire exchange from nontunnelled catheters: is there a greater risk of infection when compared with new-site replacement? Hemodial Int. 2008 12(1):52-4.

5. Coupez E, Timsit JF, Ruckly S, Schwebel C, Gruson D, Canet E, et al. Guidewire exchange vs. new site replacement for temporary dialysis catheter insertion in ICU patients: is there a greater risk of colonization or dysfunction? Crit Care. 2016;20(1):230.

6. Rabindranath KS, Kumar E, Shail R, Vaux E. Use of real-time ultrasound guidance for the placement of hemodialysis catheters: a systematic review and meta-analysis of randomized controlled trials. Am J Kidney Dis. 2011;58(6):964-70.

7. Tammam TF, El-Shafey EM, Tammam HF. Ultrasound-guided internal jugular vein access: comparison between short axis and long axis techniques. Saudi J Kidney Dis Transpl. 2013;24(4):707-13.

8. Schillinger F, Schillinger D, Montagnac R, Milcent T. Post catheterisation vein stenosis in haemodialysis: comparative angiographic study of 50 subclavian and 50 internal jugular accesses. Nephrol Dial Transplant. 1991;6(10):722-4

9. Parienti JJ, Thirion M, Megarbane B, Souweine B, Ouchikhe A, Polito A, et al. Femoral vs. jugular venous catheterization and risk of nosocomia events in adults requiring acute renal replacement therapy: a randomized controlled trial. JAMA. 2008;299(20):2413-22.

10. Parienti JJ, Megarbane B, Fischer MO, Lautrette A, Gazui N, Marin N, et al. Catheter dysfunction and dialysis performance according to vascular access among 736 critically ill adults requiring renal replacement therapy: a randomized controlled study. Crit Care Med. 2010;38(4):1118-25.

11. Dugue AE, Levesque SP, Fischer MO, Souweine B, Mira JP, Megarbane $B$, et al. Vascular access sites for acute renal replacement in intensive care units. Clin J Am Soc Nephrol. 2012;7(1):70-7.

12. Marik PE, Flemmer M, Harrison W. The risk of catheter-related bloodstream infection with femoral venous catheters as compared to subclavian and internal jugular venous catheters: a systematic review of the literature and meta-analysis. Crit Care Med. 2012;40(8):2479-85.
13. Annigeri RA, Ostermann $M$, Tolwani $A$, Vázquez-Rangel $A$, Ponce $D$, Bagga A, et al. Renal support for acute kidney injury in the developing World. Kidney Int Rep. 2017;2(4):559-78.

14. Mathur M, D'Souza AV, Prasad D, Garsa R, Bansal N, Jhorawat R, et al. A comparative study of central versus posterior approach for internal jugular hemodialysis catheter insertion. Indian J Nephrol. 2015; 25(5):265-8.

15. Morgan D, Ho K, Murray $\mathrm{C}$, Davies $\mathrm{H}$, Louw J. A randomized trial of catheters of different lengths to achieve right atrium versus superior vena cava placement for continuous renal replacement therapy. Am J Kidney Dis. 2012;60(2):272-9.

16. Engstrom BI, Horvath JJ, Stewart JK, Sydnor RH, Miller MJ, Smith TP, et al. Tunneled internal jugular hemodialysis catheters: impact of laterality and tip position on catheter dysfunction and infection rates. $\mathrm{J}$ Vasc Interv Radiol. 2013;24(9):1295-302.

17. Kam KY, Mari JM, Wigmore TJ. Adjacent central venous catheters can result in immediate aspiration of infused drugs during renal replacement therapy. Anaesthesia. 2012;67(2):115-21.

18. Contreras G, Liu PY, Elzinga L, Anger MS, Lee J, Robert N, et al. A multicenter, prospective, randomized, comparative evaluation of dual- versus triple-lumen catheters for hemodialysis and apheresis in 485 patients. Am J Kidney Dis. 2003;42(2):315-24

19. Leblanc M, Fedak S, Mokris G, Paganini EP. Blood recirculation in temporary central catheters for acute hemodialysis. Clin Nephrol. 1996; 45(5):315-9.

20. Little MA, Conlon PJ, Walshe JJ. Access recirculation in temporary hemodialysis catheters as measured by the saline dilution technique. Am J Kidney Dis. 2000;36(6):1135-9.

21. Bellomo R, Martensson J, Lo S, Kaukonen KM, Cass A, Gallagher M; RENAL study investigators; Australian and New Zealand Intensive Care Clinical Trials Group. Femoral access and delivery of continuous renal replacement therapy dose. Blood Purif. 2016;41(1-3):11-7.

22. Kim IB, Fealy N, Baldwin I, Bellomo R. Insertion side, body position and circuit life during continuous renal replacement therapy with femoral vein access. Blood Purif. 2011;31(1-3):42-6.

23. Weijmer MC, Vervloet M, Gter Wee PM. Prospective follow-up of a novel design haemodialysis catheter; lower infection rates and improved survival. Nephrol Dial Transplant. 2008;23(3):977-83.

24. Verbeke F, Haug U, Dhondt A, Beck W, Schnell A, Dietrich R, et al. The role of polymer surface degradation and barium sulphate release in the pathogenesis of catheter-related infection. Nephrol Dial Transplant. 2010;25(4):1207-13.

25. Lai NM, Chaiyakunapruk N, Lai NA, O'Riordan E, Pau WS, Saint S. Catheter impregnation, coating or bonding for reducing central venous catheter-related infections in adults. Cochrane Database Syst Rev. 2016;3:CD007878.

26. Wang Y, Ivany JN, Perkovic V, Gallagher MP, Woodward M, Jardine MJ. Anticoagulants and antiplatelet agents for preventing central venous haemodialysis catheter malfunction in patients with end-stage kidney disease. Cochrane Database Syst Rev. 2016:4:CD009631.

27. Hu HH, Hsu CY, Fang HC, Lee PT, Chen CL, Chang TY, et al. Low-dose heparin retention in temporary hemodialysis double-lumen catheter does not increase catheter occlusion and might reduce risk of bleeding. Blood Purif. 2011;32(3):232-7.

28. Zhao Y, Li Z, Zhang L, Yang J, Yang Y, Tang Y, Fu P. Citrate versus heparin lock for hemodialysis catheters: a systematic review and meta-analysis of randomized controlled trials. Am J Kidney Dis. 2014;63(3):479-90.

29. Dogra GK, Herson $\mathrm{H}$, Hutchison $B$, Irish $\mathrm{AB}$, Heath $\mathrm{CH}$, Golledge $\mathrm{C}$, et al. Prevention of tunneled hemodialysis catheter-related infections using catheter-restricted filling with gentamicin and citrate: a randomized controlled study. J Am Soc Nephrol. 2002;13(8):2133-9.

30. Ullman AJ, Cooke ML, Mitchell M, Lin F, New K, Long DA, et al. Dressings and securement devices for central venous catheters (CVC). Cochrane Database Syst Rev. 2015(9):CD010367.

31. Mimoz O, Lucet JC, Kerforne T, Pascal J, Souweine B, Goudet V, et al. Skin antisepsis with chlorhexidine-alcohol versus povidone iodine-alcohol, with and without skin scrubbing, for prevention of intravascular-catheter-related infection (CLEAN): an open-label, multicentre, randomised, controlled, two-by-two factorial trial. Lancet. 2015;386(10008): 2069-77. 